\title{
Blucher
}

Blucher Proceedings

Cuba e Brasil no Século XXI (CBS21)

Desafíos Estratégicos e Institucionais da Inovação

\section{Cidades Inteligentes, um Novo Paradigma da Sociedade do Conhecimento}

\author{
Dr. Andre L.L. Aquino, Dr. Heitor S. Ramos, \\ Dr. Leonardo V. Pereira, Dr. Alejandro C. Frery ${ }^{1}$
}

\section{Intróito}

Esse trabalho apresenta uma breve discussão a respeito de conceitos emergentes em cidades inteligentes que podem ser facilmente incorporados aos países Latinoamericanos como Brasil e Cuba. Exploramos quatro importantes temas em cidades inteligentes: mobilidade inteligente, localização de dispositivos, monitoramento de recursos naturais e prédios inteligentes. Esses temas são comumente utilizados para definir a forma em que uma cidade inteligente se comporta e cresce como um todo, quais são as suas tendências mais importantes, e qual o alcance e as oportunidades do ponto de vista da inovação e do desenvolvimento tecnológico ${ }^{2}$.

\section{Introdução}

\footnotetext{
${ }^{1}$ Instituto de Computação, Universidade Federal de Alagoas. E-mail: acfrery@gmail.com. Autor de referência Todos os demais autores do pfresente artigo também pertencem à mesma instituição do autor de referência.

${ }^{2}$ As conceituações sobre o tema apresentadas no presente texto podem ser buscadas em artigos publicados pelos autores (ver seus Currículos Lattes).
} 
Uma cidade inteligente é um sistema urbano que utiliza tecnologia de informação e comunicação para trazer mais iteratividades tanto aos aspectos de infraestrutura como aos serviços públicos em geral. Essa iteratividade visa a acessibilidade e eficiência sob o ponto de vista dos cidadãos. Ademais, é de se esperar que uma cidade inteligente esteja comprometida com o meio ambiente e com a sua herança histórica e cultural. Nesse cenário, a infraestrutura pode ser equipada com as mais avançadas soluções tecnológicas com o intuito de facilitar a interação do cidadão com os elementos urbanos.

A denominação "Cidade Inteligentes" não é usada de forma consistente na literatura. Termos semelhantes são comumente empregados: Cidades Virtuais, Cidades do Conhecimento, Cidades Digitais, ou ainda Cidades de Informação. Independente da denominação empregada todos os conceitos estão correlacionados em suas definições, aspectos técnicos (tecnologias inteligentes), de recursos humanos (gestão de pessoas inteligentes) e de governança (colaborações inteligentes). Vale destacar que concentraremos as nossas discussões nos aspectos técnicos de cidades inteligentes.

As cidades inteligentes advêm, principalmente, de dois importantes fatores. O primeiro é o aumento da população mundial aliada à crescente migração de áreas rurais para centros urbanos; estima-se que $70 \%$ da população mundial estará concentrada em áreas urbanas até 2050. O segundo fator é que existe uma preocupação com a evidente escassez de recursos naturais, que compromete a oferta global para a população mundial. Essa preocupação vem fortemente atrelada aos problemas relacionados ao meio ambiente e às constantes mudanças climáticas observadas atualmente. Isso nos remete a importantes reflexões a respeito dos novos desafios que devemos enfrentar no próximo milênio como, por exemplo, minimizar o consumo de recursos energéticos naturais, promover a utilização de energias renováveis e reduzir a emissão de $\mathrm{CO}_{2}$ na atmosfera.

O conceito de cidades inteligentes é uma ferramenta poderosa para enfrentar essas mudanças, pois elas são capazes de gerir de forma eficiente a infraestrutura e os serviços, enquanto atendem a maior parte das necessidades da cidade e seus cidadãos.

Nesse cenário, uma cidade inteligente pode ser vista como a que utiliza a tecnologia para melhorar sua infraestrutura e seus serviços, ou seja, para tornar os setores da administração, educação, saúde, segurança pública, moradia e transporte mais inteligentes, interconectados e eficientes. De fato, o conceito de cidades inteligentes pode ser visto como o reconhecimento da importância das tecnologias em geral no cotidiano das cidades. Embora existam diferentes perspectivas sobre cidades inteligentes, como citado anteriormente, a ideia de que as tecnologias em geral são fundamentais para o funcionamento futuro das cidades está no centro de todas essas perspectivas. 
Este aspecto não restringe o fato de termos que levar em consideração as questões sociais ao definirmos as cidades inteligentes. Há consenso, por exemplo, sobre a importância de termos indústrias criativas para o crescimento e a sustentabilidade urbana. O ponto principal é que a tecnologia constitui o ponto de partida para repensar todas essas outras questões.

Nos aspectos técnicos de cidades inteligentes é possível observar diversas alternativas que as novas tecnologias oferecem para o fortalecimento do sistema urbano em geral. Podemos citar as diferentes soluções para a problemática de economia dos recursos energéticos em grandes cidades, por intermédio de smart grids; a integração de veículos "inteligentes" capazes de interagir entre si para compartilhar informações de acidentes ou congestionamentos, e a utilização de sensores inteligentes e sistemas embarcados com comunicação sem fio para automação de prédios, permitindo assim a concepção de ambientes inteligentes.

Trazendo a discussão de cidades inteligentes para o contexto de países Latinoamericanos, no nosso caso Brasil e Cuba, destacaremos ao longo do texto quatro importantes temas que podem ser utilizados para definir como uma cidade se comporta e cresce como um todo, quais são as suas tendências mais importantes, e qual o alcance e as oportunidades do ponto de vista da inovação e do desenvolvimento tecnológico. Esses aspectos podem ser o ponto de partida para a caracterização de uma cidade como inteligente. Os temas que serão aqui discutidos são: mobilidade inteligente, localização de dispositivos computacionais móveis no contexto das cidades inteligentes, monitoramento inteligente de recursos naturais e prédios inteligentes.

\section{Mobilidade Inteligente}

O transporte urbano é uma importante questão na sociedade de hoje. Somos obrigados a nos mover para executar funções vitais ao avanço da sociedade. No entanto, ao observarmos as soluções de mobilidade urbana, não podemos dizer que elas formam um sistema sustentável; para isso basta observarmos os problemas causados por alterações climáticas, sem mencionar, as emissões locais de poluentes, ruído, congestionamento e acidentes. Por essa razão, estão sendo estudadas novas tecnologias de transporte e sistemas de gestão de tráfego urbano. Algumas das tendências no âmbito de cidades inteligentes são: a otimização nos serviços de localização de veículos e pessoas, a instalação inteligente de pontos de acesso para veículos em centros urbanos, os serviços de assistência aos motoristas e a detecção colaborativa de colisões e outros eventos. Uma outra vertente é a da construção de veículos autônomos que dispensam a presença de motoristas.

Com o advento de veículos com capacidade de sensoriamento e comunicação, estudos, resultados e inovações em sistemas de transportes inteligentes vêm se consolidando e sendo incorporados em cidades inteligentes. Cada vez mais, aplicações 
que fazem uso das redes veiculares ad-hoc, do inglês Vehicular Ad-Hoc Networks, tornam-se presentes e necessárias ao dia-a-dia.

Uma rede veicular é uma rede $a d$-hoc composta por veículos que possuem equipamentos de sensoriamento e comunicação sem fio e, por isso, durante o seu deslocamento, são capazes de obter informações sobre o ambiente que os rodeiam e de trocar mensagens entre si e/ou entre pontos de acessos distribuídos pela cidade.

Os dados coletados pelas redes veiculares podem oferecer, dentre outros serviços, informações sobre as condições das estradas, do tráfego e do clima, sobre o comportamento dos veículos e dos motoristas. Tais informações são úteis para uma grande variedade de aplicações: emissão de alertas de segurança, assistência ao motorista e roteamento de tráfego. Além disso, essas informações podem ser usadas para criar um sistema de tráfego inteligente capaz, dentre outras coisas, de atualizar os ciclos dos semáforos, de indicar prováveis zonas de pedágio, de medir a quantidade diária de veículos nas estradas ou de disseminar informações personalizadas aos motoristas.

Há uma série de cenários em que as redes veiculares podem ser utilizadas com sucesso. Um deles por exemplo representa uma área com a presença de poucos veículos como, por exemplo, rodovias. Outros cenários ilustram por exemplo áreas urbanas que eventualmente possuem uma maior quantidade de veículos. Em todos os casos, a informação é transmitida quando receptor e transmissor estão dentro de seus alcances de transmissão, ou quando há uma infraestrutura de comunicação disponível para ser utilizada pelos veículos.

Dentre os diferentes cenários que podem ser desdobrados podemos destacar a disseminação de informação, seja ela por intermédio de uma infraestrutura ou utilizando apenas os próprios carros. Além disso, facilmente identificamos diferentes aplicações que podem ser derivadas dessa representação dos sistemas de transportes como, por exemplo, a identificação de fluxo e a contagem e sugestão de vagas em estacionamentos.

\section{Tecnologias para redes veiculares}

O primeiro aspecto tecnológico diz respeito à disseminação de informações. Para esse caso, além dos veículos encontramos distribuídos ao longo da cidade agentes de disseminação de informações que são pontos de acessos, que ajudam a superar limitações de comunicação presentes nas redes veiculares. Nesse caso, a montagem da infraestrutura para atender a rede veicular é um aspecto importante nas cidades inteligentes. Alguns modelos teóricos foram propostos e testados no centro das cidades de Zurique e Winterthur, e nas áreas rurais de Baden e Baar, todas cidades Suiças.

Os modelos anteriormente mencionados assumem que num dado cruzamento entre duas ou mais rodovias, podemos instalar um ponto de acesso com uma área 
limitada pelo seu alcance de transmissão. Por esse cruzamento diferentes veículos podem circular, sendo que cada um possui registrado o seu tempo de permanência na interseção. Existem vários veículos circulando durante um determinado período e, para fins de instalação dos pontos de acesso, um tempo mínimo de permanência em cada ponto de disseminação é necessário para que um veículo possa receber alguma informação com sucesso. A transmissão não precisa ser realizada por um único ponto de acesso, um deles pode começar a transmissão e outros podem terminá-la, desde que o veículo permaneça em suas áreas de alcance durante o tempo mínimo exigido. Com isso, os modelos acima citados buscam instalar um número mínimo de pontos de acessos em uma cidade visando obter a maior cobertura de veículos possível. Desconsiderando a particularidade de cada um dos modelos, observamos que é possível se projetar uma cidade com um número mínimo de pontos de acesso atendendo a grande maioria dos veículos.

De forma complementar, podemos utilizar apenas os carros para disseminar informações, sem utilizar a infraestrutura. Nesse caso, a informação disseminada, geralmente, diz respeito a acidentes ou grandes congestionamentos. Ou ainda, pode ser utilizada em regiões em que não se justifica uma infraestrutura para disseminação de informação, como por exemplo, auto-estradas. Em ambos os casos, o objetivo é reportar eventos para um determinado grupo de veículos que estão em uma região específica. Para isso são propostos diferentes protocolos de disseminação de dados em rodovias, os quais são capazes de adaptar-se de forma transparente às condições de tráfego na rede de comunicação intra-veículos com o objetivo de garantir a entrega dos dados para o maior número possível de destinatários.

\section{Principais aplicações}

Em geral os engenheiros de tráfego são responsáveis por definir os intervalos de cada semáforo, o sentido das vias, e as restrições de fluxo, dentre outros parâmetros, a fim de obter a melhor fluidez do sistema evitando os congestionamentos. Entretanto, tal tarefa só é possível se eles dispuserem de dados confiáveis e atualizados a respeito do estado das vias para que possam tomar as decisões corretas. Um dos principais dados necessários é o fluxo de veículos. Nesse contexto existem aplicações em cidades inteligentes destinadas a identificar o fluxo de veículos em vias urbanas para, assim, ser possível estimar o estado de ocupação de uma via de rolamento.

Uma forma barata e escalável de identificar objetos de trânsito é o uso de etiquetas RFID (Radio-Frequency IDentification). Tais etiquetas custam poucos centavos de dólares, podem ser lidas de distâncias que variam de centímetros a metros, e não necessitam de fontes de energia. Vale salientar que a legislação brasileira já prevê a incorporação de etiquetas RFID aos carros, facilitando assim a 
implementação de sistemas como rastreamento e contagem de veículos. A contagem de veículos permite aplicações tais como identificação de congestionamentos, controle de vagas em estacionamentos, mensuração de público em eventos, e identificação de fluxo de veículos em vias, dentre outras. O rastreamento, por sua vez, permite a identificação de rotas, que podem ser utilizadas para conhecer os hábitos dos motoristas e assim melhorar o fluxo de veículos. Além disso, o rastreamento permite a identificação de congestionamentos e até mesmo a identificação de infrações por excesso de velocidade (através da análise do tempo de passagem entre dois pontos).

Outro aspecto a ser considerado em cidades inteligentes é que, devido ao aumento da frota de veículos particulares, a busca por vagas de estacionamento consome um bom tempo dos condutores. Este problema ocorre em geral em éreas urbanas, onde o número de veículos é maior do que a quantidade de vagas de estacionamento disponíveis. Para a comodidade dos condutores, os próprios estacionamentos deveriam ser responsáveis por informar a disponibilidade de vagas. No entanto, tal sistema, se realizado de maneira manual, precisaria de uma grande quantidade de recursos humanos, portanto a utilização de sistemas autônomos sem a supervisão humana para detecção de vagas têm sido implantados em diversos estacionamentos. Para esse fim, temos sistemas que utilizam de tecnologia RFID ou sensores, e sistemas que utilizam câmeras de monitoramento dedicadas ou oportunistas.

\section{Localização de Dispositivos Computacionais Móveis no Contexto das Cidades Inteligentes}

Devido à mobilidade natural dos cidadãos, a comunicação sem fio, as tecnologias de computação móvel desempenham um papel fundamental para o desenvolvimento das cidades inteligentes. Dispositivos móveis, como os telefones celulares inteligentes (smart phones), dotados de comunicação sem fio, viabilizam o acesso a serviços de utilidade pública das cidades a partir de qualquer lugar, sem a necessidade de estarem conectado a um computador convencional. Além disso, como mencionado anteriormente, veículos estão sendo dotados de comunicação sem fio e de elementos de processamento, o que os torna parte da infraestrutura de computação móvel que esá revolucionando as grandes cidades. Diversos elementos computacionais móveis, como os telefones inteligentes e os veículos, são dotados de uma grande variedade de sensores, fato que transforma toda essa infraestrutura em uma grande rede de sensores móveis que, de maneira oportunista, coleta uma grande quantidade de dados e permite a construção de diversas aplicações.

Os dados coletados por essa rede de sensores de larga escala são sensíveis à localização de onde eles foram coletados. Por exemplo, para automatização e melhoria do transporte público, a localização e o rastreamento dos veículos é de vital 
importância. Da mesma maneira, dados sobre a poluição do ar para monitoramento ambiental também devem ser acompanhados da localização geográfica de onde eles foram coletados.

Os prédios também usufruem das tecnologias de informação e comunicação nas cidades inteligentes, e formam os chamados "prédios inteligentes". Nesses ambientes, diversas tarefas são automatizadas de acordo com a localização dos indivíduos que os habitam. Assim, tarefas como controle de temperatura, eficiência energética, gerenciamento de incidentes de segurança, entre outras, podem ser realizadas com sucesso uma vez que os diversos sensores coletem dados associados com a informação da sua localização. Nesses prédios, robôs também são utilizados para automatizar diversas tarefas como limpeza, segurança, acompanhamento de visitantes, entre outras. Tanto a localização de indivíduos como a localização autônoma de robôs se tornam tarefas de suma importância para ambientes inteligentes. Assim, a localização geográfica desempenha um papel fundamental para a computação móvel, tanto em ambientes internos quanto externos.

A localização de dispositivos móveis é uma tarefa desafiadora e diversas linhas de pesquisa vem sendo realizada nesse contexto. Por exemplo, os telefones celulares do tipo smart phones são amplamente utilizado atualmente e adotam técnicas de localização baseadas em GPS (Global Positioning System). Apesar do GPS ser amplamente utilizado e produzir, em geral, pequenos erros de localização, este sensor consome uma quantidade significativa de energia e pode descarregar a bateria do dispositivo rapidamente quando utilizado por longos períodos. Algumas técnicas como as apresentadas foram propostas para tentar aliviar o alto consumo de energia do GPS, transferindo parte do processamento de sinais requerido pelas técnicas utilizadas no GPS para uma nuvem computacional. Outros autores, diferentemente, propõem evitar o uso do GPS sempre que a aplicação não necessite alta precisão, fazendo uso de sensores que consomem menos energia, como as redes de celular (Ex: 3G) e o Wireless Fidelity (WiFi). Os automóveis também fazem ampla utilização do GPS e as aplicações que irão funcionar nas redes de transportes inteligentes podem usufruir do GPS para coletar dados de localização. Porém, o GPS nem sempre está disponível, principalmente por causa da grande concentração de prédios e obstáculos que obstruem a propagação do sinal dos satélites, impedindo por vezes que esse sensor seja utilizado com sucesso. Assim, outras técnicas de localização se fazem necessárias.

Apesar de ser amplamente utilizado para localização em ambientes externos, o GPS não é adequado para ser utilizado em ambientes internos onde o sinal emitido pelos satélites raramente pode ser capturado com qualidade suficiente para serem utilizados. Dessa maneira, técnicas alternativas ao GPS devem ser propostas para essas situações. Por exemplo, autores propuseram técnicas baseadas apenas nas informações dos sensores inerciais como o acelerômetro e o giroscópio, para realizar 
a localização do dispositivo em ambientes internos. Para minimizar o acúmulo e a propagação do erro de estimação em que essa técnica conhecidamente incorre, os autores desenvolveram algoritmos que descrevem o padrão de movimentação humano e incorporaram esses padrões para correção de erros. Apesar de apresentar resultados promissores, os trabalhos publicados no tema ainda deixam alguns aspectos para serem investigados. Por exemplo, a detecção da direção de movimentação com alta precisão ainda é um problema em aberto. Nestes casos, os autores em geral não utilizam infraestrutura acoplada ao prédio para realizar a localização.

Outros autores utilizam pulsos de ultrassom para realizar a localização de dispositivos em ambientes internos. Assim, o smart phone utiliza o microfone para capturar os sinais provenientes de pelo menos três estações emissoras de ultrassom para localizar o dispositivo utilizando triangulação. Dessa maneira, essa técnica utiliza uma infraestrutura que deve estar previamente instalada no prédio. Este tipo de trabalho também apresenta problemas em aberto pois os autores em geral não tratam do problema da propagação do sinal por caminhos múltiplos, o que pode ocasionar erros grandes.

Há autores que observaram que os ambientes internos possuem assinaturas que podem ser identificadas por algum sensor conectado ao smart phone. Por exemplo, os elevadores produzem um determinado padrão de sinal no acelerômetro do celular. O padrão do sinal sonoro será diferente para uma cafeteria, por exemplo. É possível construir uma biblioteca de padrões de sonorização capturados pelo microfone do celular. Dessa maneira, se poderia caracteriz diversos ambientes e propor a utilização das assinaturas dos sinais coletados durante a caracterização dos ambientes para localizar os dispositivos.

Percebe-se que a localização de dispositivos móveis no contexto das cidades inteligentes é uma técnica básica, porém ainda carece de investimentos em pesquisa para que todas as soluções para os diversos cenários desafiadores apresentados nas cidades inteligentes sejam realizadas a contento. Essa é uma área ativa em termos de pesquisa científica, e diversas técnicas de localização no contexto de dispositivos móveis ainda apresentam espaço para investigações e melhorias.

\section{Monitoramento Inteligente de Recursos Naturais}

Cidades inteligentes têm uma ampla gama de desenvolvimento e evolução no campo da eficiência energética e da gestão de recursos. Entre essas linhas de desenvolvimento podemos encontrar: monitoramento de poluentes tanto do ar como de rios e monitoramento e alarme sobre as condições climáticas de centros urbanos. Nesse contexto podemos identificar uma variedade de fenômenos que podem ser descritos por algumas grandezas, como temperatura, pressão e umidade, que podem ser monitoradas por dispositivos com poder de sensoriamento, processamento 
e comunicação. O conjunto desses dispositivos, trabalhando de forma cooperativa, é conhecido como rede de sensores sem fios.

Uma representação bastante elegante para o processo de monitoramento inteligente de recursos naturais é apresentada pelo diagrama da figura em que $\mathcal{N}$ denota o ambiente e o processo a ser medido, e $F$ é o fenômeno de interesse, com $\mathcal{V}^{*}$ seu domínio espaço-temporal. Se uma observação foi completada sem problemas, teremos um conjunto de regras ideais $R^{*}$ para a tomada de decisões ideais $D^{*}$.

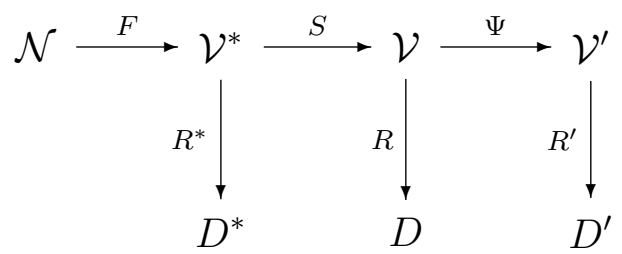

Figura 1: Representação de um sistema de uma rede de sensores onde é mostrado o comportamento ideal $\left(\mathcal{N} \rightarrow \mathcal{V}^{*} \rightarrow D^{*}\right)$, sensoriado $\left(\mathcal{N} \rightarrow \mathcal{V}^{*} \rightarrow \mathcal{V} \rightarrow D\right)$ e processado $\left(\mathcal{N} \rightarrow \mathcal{V}^{*} \rightarrow \mathcal{V} \rightarrow \mathcal{V}^{\prime} \rightarrow D^{\prime}\right)$.

Ao invés de uma situação ideal, temos um conjunto de $s$ sensores,

$$
S=\left(S_{1}, \ldots, S_{s}\right)
$$

monitorando um fenômeno e produzindo conjuntos de amostras no domínio $\mathcal{V}_{i}$, com $1 \leq i \leq s$; todos os possíveis conjuntos do domínio são denotados por $\mathcal{V}=$ $\left(\mathcal{V}_{1}, \ldots, \mathcal{V}_{s}\right)$. Usando todas essas informações, parciais e potencialmente ruidosas e incompletas, podemos conceber um conjunto de regras $R$ para prover um conjunto de decisões $D$.

Utilizar todo o conjunto $\mathcal{V}$ pode ser muito oneroso em termos de energia, largura de banda, recursos computacionais e, especialmente, no tempo de entrega das mensagens gerando atrasos para as aplicações. Uma vez que existe redundância nos dados da maioria das aplicações, o processamento dos dados pode não degradar a informação sensoriada. Esse processamento é denotado por $\Psi$, e os dados pósprocessados são denotados por $\mathcal{V}^{\prime}$. Com isso, as novas regras que usam $\mathcal{V}^{\prime}$ são denotadas por $R^{\prime}$, e elas conduzem a um conjunto de decisões $D^{\prime}$.

Com base nessa representação podemos derivar diferentes abordagens para o monitoramento inteligentes dos recursos naturais de cidades inteligentes, por exemplo, como os fenômenos são representados e caracterizados, quais as principais técnicas de processamento de dados podem ser utilizados ou como garantir que as decisões tomadas pelos gestores são satisfatórias, por exemplo, a decisão de evacuar uma áarea prestes a ser alagada. Nesse contexto descreveremos algumas dessas abordagens. 
Dentre as diferentes estratégias para o processamento dos dados em redes de sensores existem algumas abordagens que propõem a utilização de amostragem adaptativa, ou seja, à medida que o fenômeno monitorado se modifica, a forma de amostragem se adapta para obter dados mais precisos. Nesse caso, geralmente, os dados são processados com o objetivo de identificar suas correlações e assim eliminar redundância. De forma complementar, outras abordagens realizam o processamento com técnicas como fusão, agregação, compressão, correlação ou redução de dados, todas baseadas na correlação das informações e aplicadas com o objetivo de economizar recursos da rede, como energia, tempo de resposta e perda de pacotes.

\section{Principais aplicações}

Uma antiga e interessante aplicação está relacionada ao projeto "Great Duck Island", que tinha por objetivo monitorar o comportamento e o habitat do pássaro chamado "Storm Petrel". Inicialmente, trinta-e-dois nós sensores foram colocados nas tocas dos pássaros e também próximos a elas. Posteriormente, a rede foi aumentada, ganhando mais nós e estações meteorológicas. Os nós sensores podiam medir a temperatura, a umidade do ar e a pressão barométrica, dentre outras variáveis. As leituras periódicas dos nós eram transmitidas a um observador externo através da comunicação entre os nós. O observador externo retransmitia as leituras até a estação base, onde eram armazenadas. Uma réplica do banco de dados era transmitida, via satélite, para um servidor na Universidade de Berkeley a cada quinze minutos.

Outra aplicação similar é a "ZebraNet", que utiliza colares sensores equipados com GPS instalados em zebras na reserva de Sweetwaters no Quênia. Do ponto de vista biológico, o objetivo do projeto é monitorar o comportamento noturno dos animais e ainda responder questões acerca de migração e relacionamento interespécies. Os sensores foram projetados para registrarem e armazenarem a posição dos animais a cada três minutos. A cada hora, outros sensores registram por três minutos dados meteorológicos, ambientais, de iluminação, de temperatura e de movimentos corporais. A estação base móvel percorre o campo dos sensores, habitat das zebras, para recolher os dados. Ambas aplicações podem ser consideradas no contexto de cidades inteligentes para o monitoramento de animais em zoológicos públicos ou para o monitoramento e rastreamento de animais domésticos.

Uma aplicação de bastante importância para cidades inteligentes é o monitoramento da qualidade do ar. Nessas aplicações os dados utilizados são ditos multivariados, pois representam mais de um fenômeno monitorado simultaneamente; por exemplo, os dados monitorados por uma estação que possui simultaneamente sensores de temperatura, gás carbônico, óxido de nitrogênio, dióxido de nitrogênio entre outros. Algumas cidades brasileiras, como Rio de Janeiro e São Paulo, 
possuem estações de monitoramento que usam sensores para obter informações a respeito da qualidade do ar. Nessas aplicações, a qualidade do ar é monitorada em inúmeras áreas através da medição de diferentes parâmetros. Como diferentes variáveis são monitoradas, nesse caso, as soluções utilizam o processamento de dados multivariados para se obter uma representação coerente dos dados.

\section{Prédios Inteligentes}

Nas cidades inteligentes, os cidadãos são a chave para o desenvolvimento da cidade. Nesse sentido, aspectos de conforto e bem-estar são pontos explorados nos prédios inteligentes. Dentre as características principais de prédios inteligentes que visam uma melhoria na qualidade de vida podemos destacar: monitoramento de pessoas enfermas, segurança individual, monitoramento e controle da habitação e otimização do consumo dos recursos energéticos e hidráulicos.

Um ponto chave na definição de espaços inteligentes é que eles devem possuir uma estrutura especial que permita aos ocupantes controlarem, programarem automaticamente ou que o próprio ambiente se adapte autonomamente ao comportamento dos ocupantes. "Inteligente" é viver bem, com economia, usufruindo de todos os recursos que a tecnologia atual e futura pode nos proporcionar. Há um certo tempo já temos ao nosso dispor vários recursos com um mínimo de automação embutida. Por exemplo, os sistemas de calefação, ar-condicionado e aquecimento de água já podem ser pré-programados por intermédio de um painel de controle ou até de nossos smartphones. Além disso, vários sistemas de segurança, monitoramento e iluminação podem ser programados via um simples browser.

No entanto, o sonho de consumo é que estes apetrechos possam identificar de forma autônoma nosso comportamento para se ajustarem às nossas necessidades. Nesse cenário, alguns efeitos desses espaços inteligentes seriam: a criação de cenários diferenciados nos mesmos espaços com iluminação alternada; regar o jardim e controlar a iluminação externa de uma residência; rastreamento e identificação das pessoas presentes na casa por intermédio de câmeras; ligar automaticamente a televisão no horário de seu programa predileto; ou controlar os sistemas de calefação ou ar-condicionado deixando os ambientes na temperatura que você considera ideal.

\section{Tecnologias para prédios inteligentes}

A concepção de ambientes inteligentes acarreta sérios desafios tecnológicos que englobam desde novos sensores e dispositivos embarcados até aplicações que executem em um browser ou em um smartphone. Nesses ambientes, de forma geral, identificamos a necessidade da utilização de dispositivos e sistemas embarcados 
para permitir a automatização e gerenciamento, por exemplo, da energia elétrica consumida em uma casa ou prédio. Em uma primeira instância temos as tecnologias de sensores e atuadores que são a base para todo o processo de automação inteligente de ambientes. Num segundo patamar encontramos os sistemas dedicados ou embarcados que utilizam as informações sensoreadas para controlar os diferentes equipamentos ou dispositivos eletro-eletrônicos utilizados no prédio (televisores, ar-condicionado, lâmpadas, etc). Por fim, é necessário a utilização de um sistema para gerenciar e monitorar todo consumo do prédio e, quando possível, efetuar o controle no ambiente para fins de economia de recursos.

De forma complementar, outro aspecto importante e sempre considerado nesses ambientes inteligentes são os recursos voltados à segurança de prédios e dos cidadãos. Essas aplicações fazem uso, por exemplo, das tecnologias baseadas em biometria para reconhecimento e identificação das pessoas que circulam nos prédios, câmeras inteligentes para rastreamento de pessoas e crachás inteligentes, baseados no conceito de "RFID", que permitem identificar seu portador em qualquer ambiente do prédio.

Considerando as tecnologias gerais para edifícios inteligentes temos pesquisas voltadas ao projeto de sistemas de energia alimentados por placas de captação solar integrados para conceber edifícios verdes (que se preocupam com o meio ambiente). Os principais serviços integrados são aquecimento, ar condicionado, ventilação natural e água quente. No projeto de edifícios verdes alguns aspectos específicos são considerados, como por exemplo, a avaliação da eficiência do sistema energético e da qualidade de energia.

Em alguns estudos, em geral, um aspecto de edifícios inteligentes considerado é a estrutura do edifício. O objetivo em geral é evitar catástrofes ambientais, por exemplo, terremotos, através de um sistema de controle. Os aspectos específicos considerados são o desenvolvimento de modelos de análise do sistema de isolamento e algoritmos de controle para a operação do sistema.

\section{Principais Aplicações}

Um caso de sucesso que trata da problemática de edifícios inteligentes é conhecido como "HOBNET", que procura facilitar e maximizar a utilização de plataformas denominadas de "FIRE" (Future Internet Research) ${ }^{3}$, tendo como objetivo específico a automação e eficiência energética para edifícios inteligentes/verdes. $\mathrm{O}$ projeto lida com a parte algorítmica, de redes e os aspectos de desenvolvimento de aplicações de sistemas da Internet do Futuro de pequenos dispositivos embutidos. Toda infraestrutura dos edifícios utiliza "IPv6/6LoWPAN", de forma a integrar tecnologias heterogêneas (sensores, atuadores, dispositivos móveis, etc).

\footnotetext{
${ }^{3}$ http : //cordis.europa.eu/fp7/ict/fire/
} 
Com isso, o 6lowApp e a sua padronização são propostos como um novo protocolo de aplicações integradas para automação de edifícios. A premissa principal é o desenvolvimento rápido e a integração de aplicações de gerenciamento de edifícios com base na implantação e monitoramento de aplicações resultantes dos testbeds "FIRE".

Outra aplicação bastante considerada é a utilização de robôs serviçais (recepcionistas ou assistentes) em prédios inteligentes. As principais funcionalidades que um robô serviçal deve fornecer são localização, navegação, reconhecimento de objetos e manipulação de objetos. O robô serviçal executa todas essas operações utilizando seus próprios sensores e atuadores combinados com uma base de dados de conhecimento. Outra alternativa é combinar sensores da própria casa para poder aumentar a quantidade de tarefas destinadas aos robôs. Este ambiente é constituído por objetos inteligentes com uma identificação por rádio frequência (RFID) e aparelhos inteligentes com funcionalidades de rede de sensores; um servidor doméstico que conecta dispositivos inteligentes e mantêm informações para serviços confiáveis; e os robôs serviçais que executam tarefas em colaboração com o meio ambiente.

\section{Conclusão}

Esse texto apresenta uma breve discussão a respeito de conceitos emergentes de cidades inteligentes que podem ser facilmente incorporadas nos países Latinoamericanos, como Brasil e Cuba. Foram apresentados quatro importantes temas utilizados para definir como uma cidade se comporta e cresce como um todo, quais são as suas tendências mais importantes, e qual o alcance e as oportunidades do ponto de vista da inovação e do desenvolvimento tecnológico.

O primeiro tema discutido estava relacionado à mobilidade inteligente, em que apresentamos questões relacionadas à montagem de uma infraestrutura de acesso a informações por parte dos veículos que circulam em uma determinada cidade ou a utilização dos próprios veículos conversando entre si para trocar informações e experiências sobre o trânsito.

De forma complementar, discutimos sobre a importância da localização de dispositivos móveis no contexto das cidades inteligentes e alguns desafios que essa área ainda proporciona.

Em seguida falamos sobre o monitoramento inteligente de recursos naturais, onde apresentamos como os fenômenos podem ser representados e caracterizados, quais as principais técnicas de processamento de dados podem ser utilizadas e como garantir que as decisões tomadas pelos gestores sejam satisfatórias.

Por fim, versamos sobre prédios inteligentes, onde discutimos alguns elementos que podem ser utilizados para melhorar nosso conforto doméstico pela criação de 
espaços inteligentes ou como efetuar a vigilância de prédios por intermédio de elementos tecnológicos. Além disso, apresentamos aplicações que podem facilmente ser incorporadas em cenários latinoamericanos, como a montagem de casas inteligentes e a utilização de robôs serviçais.

Recife, Olinda, Maceió, 2014 